\title{
Life expectancy in England: variations and trends by gender, health authority, and level of deprivation
}

\author{
Veena Soni Raleigh, Victor A Kiri
}

\begin{abstract}
Study objectives-To investigate variations and trends in life expectancy in English district health authorities in relation to gender and Jarman deprivation level. Design-Mortality data for English health authorities from 1984-94, compiled by the Office for National Statistics, were assessed conventionally and using life table techniques.

Setting-District health authorities in England.

Main outcome measures-Life expectancies in the 105 DHAs in relation to rank, to gender, and to deprivation category based on the census based Jarman score.

Conclusions-Differences in life expectancy had widened over the decade and prosperous areas with greatest longevity had seen the largest gains. In most deprived areas improvements in life expectancy were negligible. The greatest gender differences in life expectancy were also seen in deprived areas.
\end{abstract}

\section{(F Epidemiol Community Health 1997;51:649-658)}

The Health of the Nation white paper noted that effective strategies to improve health need to be sensitive to variations in health. ${ }^{1}$ In a recent report on health variations, the Chief Medical Officer's Working Group concluded that action to tackle these is central to the achievement of the Government's Health of the Nation strategy. ${ }^{2}$ It went on to specify actions that the Department of Health and the NHS can take to tackle the socioeconomic, regional, gender, and ethnic variations in health which prevail in the UK. Recommendations included the need for further research, including work on population groups at risk of poor health.

We examine the effects of regional variations in mortality on longevity. Mortality differences are conventionally assessed in terms of epidemiological measures such as standardised mortality rates/ratios that offer comparisons against a national average but give no tangible indication of their impact on longevity. Life table techniques convert mortality rates into life expectancy, a readily comprehensible summary index of mortality experience. Life expectancy data are available nationally, ${ }^{3}$ for regional health authorities, ${ }^{45}$ and for "clusters" of homogeneous areas ${ }^{5}$ but have not hitherto been examined for health authorities.
Using mortality data for the decade 198494, we examined variations and trends in life expectancy at birth in relation to gender in district health authorities (DHAs) in England, using recent (1995) DHA boundaries. Life expectancy was also computed for "families" of DHAs grouped on the basis of 1991 censusbased Jarman deprivation scores. ${ }^{67}$ Gender differences and trends in life expectancy over the decade 1984-94 were also examined by DHA and level of deprivation. The findings depict regional, socioeconomic, and gender inequalities in mortality in terms of a yardstick everyone can comprehend-how long men and women live.

\section{Methods}

The analysis is based on mortality data for England for the individual years 1984-94 compiled by the Office for National Statistics (ONS), and ONS final mid-year population estimates for the years 1984-94. Abridged life tables were constructed by sex using the method described by Elandt-Johnson and Johnson. ${ }^{8}$ The age groups used were $<1,1-4$, $5-9, \ldots, 80-84,85+$. Life expectancy at birth was computed for the three year period 1992-94 (three year averages were used to smooth out annual fluctuations at local level). It was also computed for 1984-86 to examine the absolute change in values between the start and end periods of the decade, and area rankings in the two periods. The SD and coefficient of variation were computed for the two periods to examine whether or not regional differences had altered. Trends in life expectancy were calculated using the methodology for computing mortality trends in the Public Health Common Data Set, produced annually by the Department of Health. ${ }^{7}$ Estimates of the average annual percent change were calculated using the logarithm of the annual life expectancies, on the basis of the formula $100\left(e^{b}-1\right)$ where $b$ is the coefficient of the slope of the resultant regression line. A goodness of fit test showed strong evidence for a linear relationship ( $p<0.01$ in all but 5 DHAs, and $p$ value $<0.05$ in the remaining 5 DHAs).

Life expectancies in 1992-94 in the 105 DHAs in England (boundaries as of April 1995) are presented by sex in rank order from highest to lowest. Values and ranks for 1984-86 are also given, along with the average annual percentage change in life expectancy during 1984-94. In the maps and graphs similar scales 
and/or intervals are used for males and females to facilitate comparison by gender.

DHAs were also grouped into seven categories on the basis of their Jarman deprivation scores, which are derived from a number of 1991 census based demographic and socioeconomic variables. ${ }^{67}$ The basis for the grouping, and the DHAs in each group, are shown in table 1. Life expectancy data for the deprivation categories are presented in the same format as described above for DHAs. Life expectancies and Jarman scores for the individual DHAs were correlated to measure the association between deprivation and longevity. Gender differences and trends in life expectancy between 1984-94 were examined by deprivation level.

\section{Results}

LIFE EXPECTANCY AND TRENDS IN DHAS

In 1992-94 life expectancy at birth in England was 74.1 years for men and 79.5 years for women (table 2). With the exception of males in Solihull (West Midlands), the DHAs with the highest life expectancies were in the south, east, or west of England. Life expectancy in both 1984-86 and 1992-94 was lowest in Manchester in both sexes. Given the mortality rates prevailing in 1992-94, men and women in Manchester die 4.2 years and 2.8 years earlier respectively than the national averages. The contrast is greater when compared with DHAs where life expectancy is highest: men and women in DHAs with the highest life expectancy live on average 6.7 years and 4.7 years longer respectively than men and women in Manchester. Other DHAs with low life expectancies at the start and end of the decade include Liverpool, Sunderland, South of Tyne, and St Helens and Knowsley. The north-south gradient in life expectancy is clearly apparent in figure 1. Exceptions to this geographical divide are the inner London DHAs of South East London, East London and the City, and Camden and Islington, where male life expectancies are among the lowest in the country.

If geographical differentials in mortality had narrowed over the decade, trends in life expectancy (fig 2) could be expected to show a gradient in the opposite direction to that in figure 1-that is, greater change in areas with low life expectancy. Such a gradient is not universally apparent, and in many DHAs low life expectancy at the start of the decade was followed by little improvement.

These regional contrasts can be seen clearly in table 2 . The annual increase in life expectancy in England over the decade was $0.35 \%$ in males and $0.25 \%$ in females. In Manchester life expectancy increased by only $0.10 \%$ in males (the lowest increase nationally) and by $0.22 \%$ in females. This was significantly lower than the corresponding figures, for instance, for Eastern Surrey $(0.45 \%$ and $0.33 \%)$. Liverpool and parts of inner London (South East London, East London and the City, Camden and Islington) similarly experienced much less improvement, particularly in men, than some DHAs with the highest life expectancies.

The high-low difference in life expectancy between DHAs increased over the decade. In
KEY POINTS

- Life expectancy in health authorities in England varies by 6.7 years in males and 4.7 years in females.

- These differences have widened over the past decade.

- Deprivation (Jarman scores) is correlated with both levels and trends in life expectancy.

- Prosperous areas with the greatest longevity have seen the largest gains in life expectancy.

- Despite having the shortest life spans in the 1980s, most deprived areas (inner London, Manchester, and Liverpool) have experienced negligible improvements.

- Deprived areas have the greatest gender differences in life expectancy.

1984-86 the difference between the highest and lowest ranking DHAs was 5.2 years for males and 4.3 years for females; by 1992-94 the difference had widened by 1.5 years $(29 \%)$ in males to 6.7 years, and by half a year $(9 \%)$ in females to 4.7 years. Furthermore, the spread of the DHA values around the national average has not decreased over the decade. The SD for males was 1.24 years in $1984-86$ and 1.34 years in 1992-94, and for females it was 1.05 and 1.10 years respectively, representing an increasing coefficient of variation from $1.72 \%$ to $1.81 \%$ in males and from $1.36 \%$ to $1.39 \%$ in females (although some of this could be due to random variation).

\section{LIFE EXPECTANCY AND TRENDS BY LEVEL OF} DEPRIVATION

Life expectancy by sex in DHAs grouped according to the level of deprivation is shown in table 3 and figure 3 . Although there is considerable overlap in the life expectancy of DHA outliers in the different deprivation categories, the negative association between deprivation and life expectancy at DHA level is evident in a correlation coefficient of -0.77 for men and a somewhat weaker association of -0.56 for women. In 1992-94 life expectancy in the most affluent areas exceeded that in the most deprived areas, which include inner London, by 4.0 years in males and 2.4 years in females. These differences had increased over those prevailing in the mid-1980s (2.8 years and 1.6 years respectively). Despite having the highest life expectancy in the mid-1980s, the most affluent areas experienced significantly greater gains in life expectancy over the decade $(0.38 \%$ in males and $0.28 \%$ in females) than the most deprived areas where life expectancy was lowest $(0.18 \%$ and $0.17 \%$ respectively).

The percentage change between 1984-86 and 1992-94 in deaths occurring up to specific ages in a cohort of 100000 births was analysed by level of deprivation. In both sexes and all age groups there was a consistent negative association between the rate of mortality decline and the level of deprivation. This effect was particularly marked for the most deprived areas, where 
Table 1 farman score based area classification using the distribution of district health authority scores (mean 0.00, (SD 17.37))

\begin{tabular}{|c|c|c|c|c|}
\hline \multirow{2}{*}{$\begin{array}{l}\text { Group } \\
\begin{array}{l}\text { Deprivation level } 1 \text { (scores }<-S D \text { (ie } \\
<-17.37)\end{array}\end{array}$} & \multicolumn{4}{|c|}{ District health authority } \\
\hline & $\begin{array}{l}\text { D09 } \\
\text { E05 } \\
\text { E18 } \\
\text { G12 } \\
\text { H20 } \\
\text { H21 } \\
\text { J25 } \\
\text { K24 }\end{array}$ & $\begin{array}{l}\text { Huntingdon } \\
\text { North West Hertfordshire } \\
\text { East and North Hertfordshire } \\
\text { Bromley } \\
\text { Western Surrey } \\
\text { Eastern Surrey } \\
\text { North and Mid Hampshire } \\
\text { Buckinghamshire }\end{array}$ & $\begin{array}{l}\text { M04 } \\
\text { M20 } \\
\text { M26 } \\
\text { M27 } \\
\text { M28 } \\
\text { N18 } \\
\text { P22 }\end{array}$ & $\begin{array}{l}\text { Worcester } \\
\text { Solihull } \\
\text { North Worcestershire } \\
\text { South Staffordshire } \\
\text { Warwickshire } \\
\text { South Cheshire } \\
\text { South Lancashire }\end{array}$ \\
\hline $\begin{array}{l}\text { Deprivation level } 2 \text { (scores between }-1.0 \text { and } \\
-0.5 \text { of the SD) }\end{array}$ & $\begin{array}{l}\text { B21 } \\
\text { C01 } \\
\text { C14 } \\
\text { C15 } \\
\text { D01 } \\
\text { D13 } \\
\text { E06 } \\
\text { F31 } \\
\text { F32 } \\
\text { G23 } \\
\text { H19 }\end{array}$ & $\begin{array}{l}\text { North Yorkshire } \\
\text { North Derbyshire } \\
\text { North Nottinghamshire } \\
\text { Lincolnshire } \\
\text { Cambridge } \\
\text { East Norfolk } \\
\text { South West Hertfordshire } \\
\text { North Essex } \\
\text { South Essex } \\
\text { West Kent } \\
\text { Kingston and Richmond }\end{array}$ & $\begin{array}{l}\text { J30 } \\
\text { K13 } \\
\text { K33 } \\
\text { K41 } \\
\text { L40 } \\
\text { L51 } \\
\text { M02 } \\
\text { M05 } \\
\text { M18 } \\
\text { P16 }\end{array}$ & $\begin{array}{l}\text { Wiltshire and Bath } \\
\text { Berkshire } \\
\text { Northamptonshire } \\
\text { Oxfordshire } \\
\text { Gloucestershire } \\
\text { Somerset } \\
\text { Herefordshire } \\
\text { Shropshire } \\
\text { Dudley } \\
\text { Stockport }\end{array}$ \\
\hline $\begin{array}{l}\text { Deprivation level } 3 \text { (scores between }-0.5 \text { and } \\
0.0 \text { of the SD) }\end{array}$ & $\begin{array}{l}\text { A11 } \\
\text { A34 } \\
\text { C02 } \\
\text { C03 } \\
\text { D05 } \\
\text { D12 } \\
\text { D14 } \\
\text { E07 } \\
\text { E09 } \\
\text { H09 } \\
\text { H22 }\end{array}$ & $\begin{array}{l}\text { Northumberland } \\
\text { North Cumbria } \\
\text { Southern Derbyshire } \\
\text { Leicestershire } \\
\text { North West Anglia } \\
\text { Suffolk } \\
\text { Bedfordshire } \\
\text { Barnet } \\
\text { Hillingdon } \\
\text { Croydon } \\
\text { West Sussex }\end{array}$ & $\begin{array}{l}\mathrm{J} 10 \\
\mathrm{~J} 21 \\
\mathrm{~J} 22 \\
\mathrm{~L} 10 \\
\mathrm{~L} 21 \\
\mathrm{~L} 35 \\
\text { M07 } \\
\text { N17 } \\
\text { N43 } \\
\text { P28 }\end{array}$ & $\begin{array}{l}\text { Dorset } \\
\text { Portsmouth and SE Hampshire } \\
\text { Southampton and SW Hampshire } \\
\text { Bristol } \\
\text { Cornwall and Isles of Scilly } \\
\text { Exeter and North Devon } \\
\text { North Staffordshire } \\
\text { North Cheshire } \\
\text { Sefton } \\
\text { Morecambe Bay }\end{array}$ \\
\hline $\begin{array}{l}\text { Deprivation level } 4 \text { (scores between } 0.0 \text { and } 0.5 \\
\text { of the SD) }\end{array}$ & $\begin{array}{l}\text { A30 } \\
\text { A31 } \\
\text { B11 } \\
\text { B16 } \\
\text { B71 } \\
\text { C08 } \\
\text { C09 } \\
\text { C10 } \\
\text { C11 } \\
\text { E19 } \\
\text { F33 }\end{array}$ & $\begin{array}{l}\text { North Durham } \\
\text { South Durham } \\
\text { East Riding } \\
\text { Grimsby and Scunthorpe } \\
\text { Wakefield } \\
\text { Nottingham } \\
\text { Barnsley } \\
\text { Doncaster } \\
\text { Rotherham } \\
\text { Brent and Harrow } \\
\text { Barking and Havering }\end{array}$ & $\begin{array}{l}\text { G21 } \\
\text { G22 } \\
\text { J41 } \\
\text { L36 } \\
\text { M21 } \\
\text { N31 } \\
\text { N51 } \\
\text { P20 } \\
\text { P23 } \\
\text { P24 }\end{array}$ & $\begin{array}{l}\text { East Sussex } \\
\text { East Kent } \\
\text { Isle of Wight } \\
\text { Plymouth and Torbay } \\
\text { Walsall } \\
\text { St Helens and Knowsley } \\
\text { Wirral } \\
\text { North West Lancashire } \\
\text { Wigan and Bolton } \\
\text { Bury and Rochdale }\end{array}$ \\
\hline $\begin{array}{l}\text { Deprivation level } 5 \text { (scores between } 0.5 \text { and } 1.0 \\
\text { of the SD) }\end{array}$ & $\begin{array}{l}\text { A16 } \\
\text { A32 } \\
\text { A33 } \\
\text { B51 } \\
\text { B61 } \\
\text { C12 } \\
\text { F34 }\end{array}$ & $\begin{array}{l}\text { Sunderland } \\
\text { Tees } \\
\text { South of Tyne } \\
\text { West Yorkshire } \\
\text { Leeds } \\
\text { Sheffield } \\
\text { Redbridge and Waltham Forest }\end{array}$ & $\begin{array}{l}\text { G24 } \\
\text { H17 } \\
\text { M17 } \\
\text { M22 } \\
\text { P21 } \\
\text { P25 } \\
\text { P27 }\end{array}$ & $\begin{array}{l}\text { Bexley and Greenwich } \\
\text { Merton, Sutton and Wandsworth } \\
\text { Coventry } \\
\text { Wolverhampton } \\
\text { East Lancashire } \\
\text { West Pennine } \\
\text { Salford and Trafford }\end{array}$ \\
\hline $\begin{array}{l}\text { Deprivation level } 6 \text { (scores between } 1.0 \text { and } \\
1.96 \text { of the SD) }\end{array}$ & $\begin{array}{l}\text { A35 } \\
\text { B31 } \\
\text { E20 } \\
\text { E21 }\end{array}$ & $\begin{array}{l}\text { Newcastle and North Tyneside } \\
\text { Bradford } \\
\text { Ealing, Hammersmith and Hounslow } \\
\text { Kensington, Chelsea and Westminster }\end{array}$ & $\begin{array}{l}\text { F36 } \\
\text { M19 } \\
\text { M25 } \\
\text { M29 }\end{array}$ & $\begin{array}{l}\text { New River } \\
\text { Sandwell } \\
\text { South Birmingham } \\
\text { North Birmingham }\end{array}$ \\
\hline $\begin{array}{l}\text { Deprivation level } 7 \text { (scores significantly greater } \\
\text { than the mean at } 5 \% \text { level (ie }>=1.96 \mathrm{SD} \text { ) }\end{array}$ & $\begin{array}{l}\text { F35 } \\
\text { F37 } \\
\text { G26 }\end{array}$ & $\begin{array}{l}\text { East London and the City } \\
\text { Camden and Islington } \\
\text { South East London }\end{array}$ & $\begin{array}{l}\text { N21 } \\
\text { P26 }\end{array}$ & $\begin{array}{l}\text { Liverpool } \\
\text { Manchester }\end{array}$ \\
\hline
\end{tabular}

by the age of 40 men had lost all improvements in survival over the decade. Mortality changes within selected age groups in a cohort of 100000 births were also analysed and showed that mortality in men aged 30-39 increased nationally and in all area groupings, with an increase of over $20 \%$ in the most deprived areas. Deprived areas also saw a significant increase in mortality among men aged 20-29. In most deprived areas, mortality increased also in women aged 10-19 and 20-29.

\section{GENDER DIFFERENCES AND TRENDS IN LIFE}

EXPECTANCY

Female life expectancy in England in 1992-94 exceeded male life expectancy by 5.4 years (table 2), however, regional differences are apparent in this respect also (fig 4). The gender differential in 1992-94 was smaller in DHAs with relatively high life expectancy, such as Cambridge (4.6 years) and Western Surrey (4.7), and greater in DHAs with relatively low life expectancy, such as Camden and Islington (7.0), Manchester (6.8), South East London (6.7), East London and the City (6.4), and Liverpool (6.1). Gender differences ranged from 5.0 years in the most affluent areas to 6.6 years in the most deprived areas (table 3 ). The strong positive association between deprivation and gender differences in life expectancy at DHA level is apparent in figure 4 , and is reflected in a correlation coefficient of 0.8 .

Furthermore, there was an association between deprivation and trends in gender differences in life expectancy. In England overall, the female-male difference in life expectancy narrowed somewhat from 5.83 years in $1984-86$ to 5.38 years in $1992-94$, a decline of 
Greater Manchester

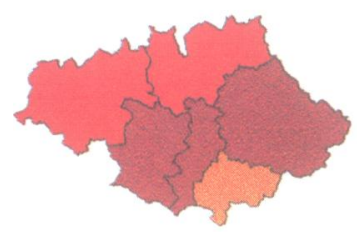

West Midlands

Metropolitan County

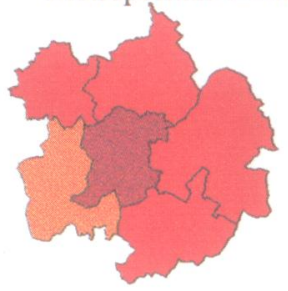

Greater London

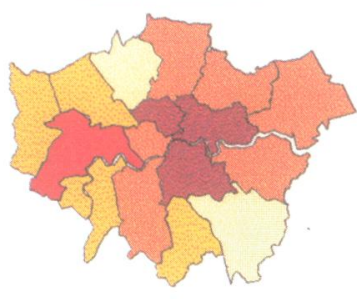

Greater Manchester

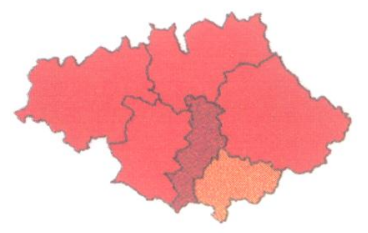

West Midlands

Metropolitan County

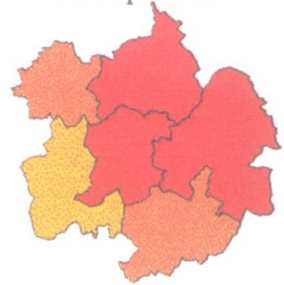

Greater London

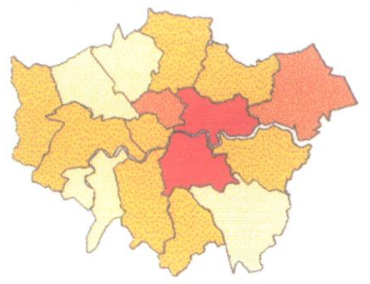

Men

Life expectancy in years

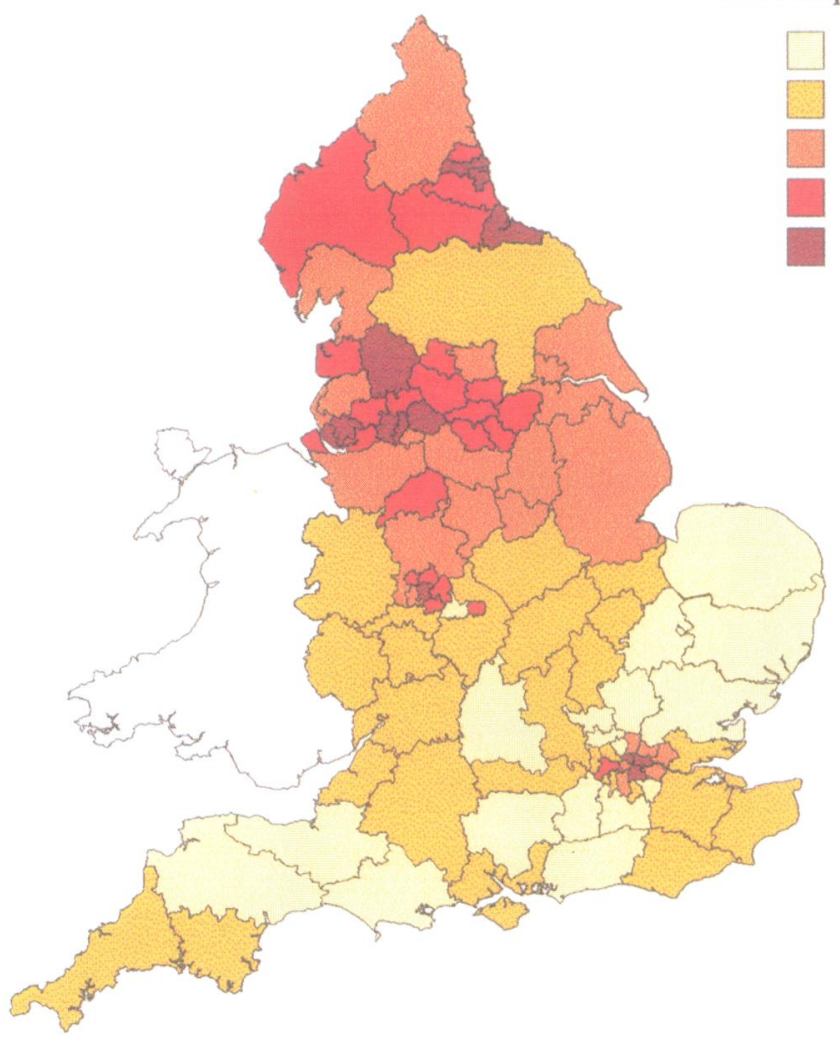

75.5 to 76.6

74.5 to 75.4

73.5 to 74.4

72.5 to 73.4

$<72.5$

Figure 1 Life expectancy (in years) among men and women in English district health authorities, 1992-94.

Life expectancy in years

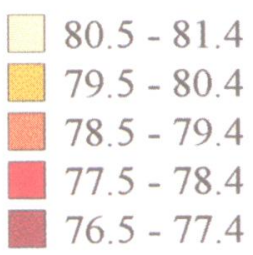

Women

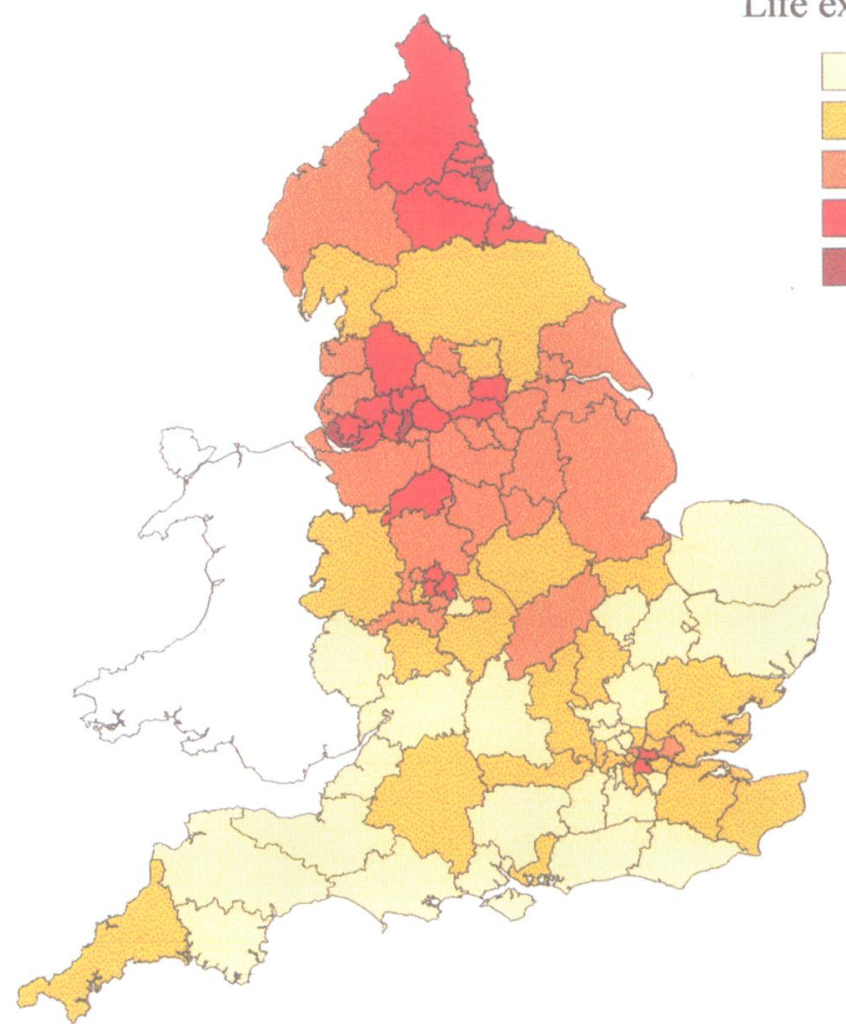


Greater Manchester

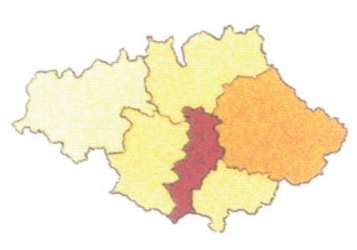

West Midlands Metropolitan County

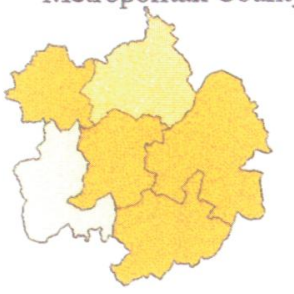

Greater London

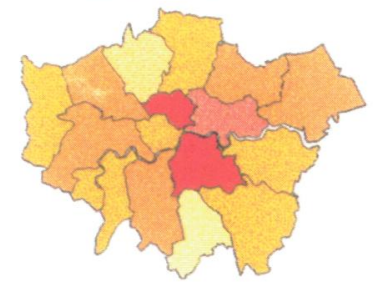

Men

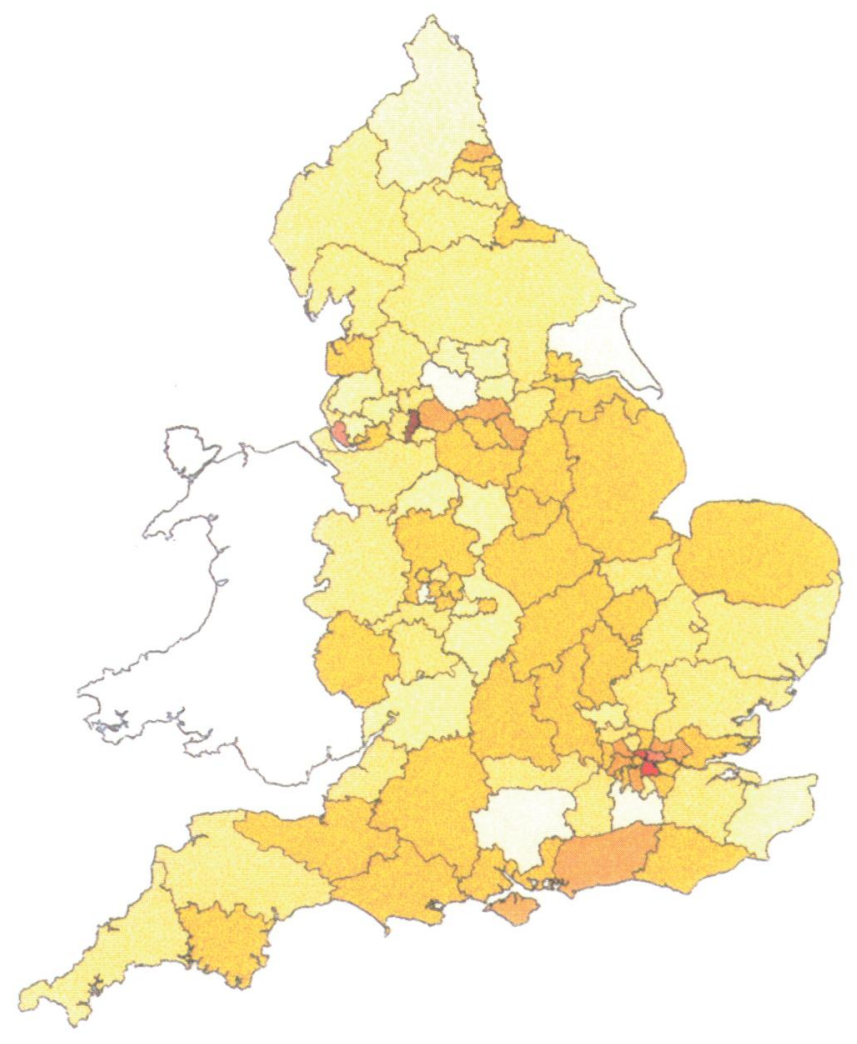

\section{Percent}

Women

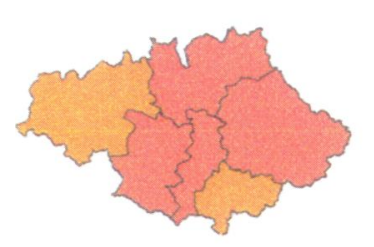

West Midlands

Metropolitan County

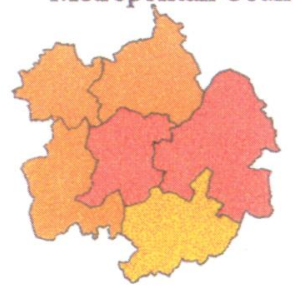

Greater London

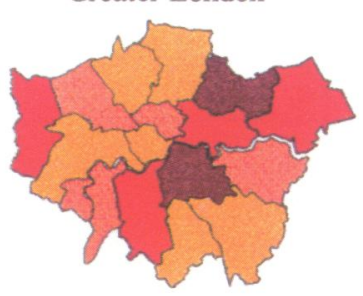

Percent

$\square 0.45-0.49$

$0.40-0.44$

$0.35-0.39$

$0.30-0.34$

$0.25-0.29$

$0.20-0.24$

$0.15-0.19$

$0.10-0.14$ 
Table 2 Life expectancy at birth in relation to gender in 1984-86 and 1992-94: English district health authorities ranked highest to lowest on the 1992-94

\begin{tabular}{|c|c|c|c|c|c|c|c|c|c|c|c|}
\hline \multirow[b]{2}{*}{ Males } & & \multirow{2}{*}{$\begin{array}{l}\text { Ranks } \\
\text { in } \\
1984-86\end{array}$} & \multicolumn{2}{|c|}{ Life expectancy } & \multirow{2}{*}{$\begin{array}{l}\% \\
\text { annual } \\
\text { increase }\end{array}$} & \multirow{2}{*}{\multicolumn{2}{|c|}{ Females }} & Ranks & Life expec & tancy & \\
\hline & & & $1984-86$ & $1992-94$ & & & & $1984-86$ & $1984-86$ & $1992-94$ & increase \\
\hline $\mathrm{O} 00$ & England & & 72.1 & 74.1 & 0.35 & $\mathrm{O} 00$ & England & & 77.9 & 79.5 & 0.25 \\
\hline D01 & Cambridge & 1 & 74.4 & 76.6 & 0.37 & G12 & Bromley & 2 & 79.6 & 81.4 & 0.28 \\
\hline $\mathrm{H} 20$ & Western Surrey & 2 & 74.2 & 76.4 & 0.37 & $\mathrm{~J} 10$ & Dorset & 3 & 79.5 & 81.4 & 0.26 \\
\hline $\mathrm{H} 21$ & Eastern Surrey & 15 & 73.4 & 76.2 & 0.45 & D01 & Cambridge & 1 & 79.7 & 81.1 & 0.24 \\
\hline E07 & Barnet & 5 & 73.9 & 76.0 & 0.36 & $\mathrm{H} 20$ & Western Surrey & 23 & 78.9 & 81.1 & 0.33 \\
\hline M20 & Solihull & 16 & 73.4 & 76.0 & 0.40 & L35 & Exeter and North Devon & 17 & 79.0 & 81.0 & 0.30 \\
\hline E05 & North West Hertfordshire & 20 & 73.4 & 75.9 & 0.43 & L51 & Somerset & 34 & 78.6 & 80.9 & 0.38 \\
\hline K41 & Oxfordshire & 3 & 74.0 & 75.9 & 0.32 & $\mathrm{H} 22$ & West Sussex & 7 & 79.3 & 80.9 & 0.25 \\
\hline $\mathrm{J} 10$ & Dorset & 4 & 73.9 & 75.9 & 0.32 & K41 & Oxfordshire & 8 & 79.2 & 80.9 & 0.27 \\
\hline G12 & Bromley & 6 & 73.8 & 75.7 & 0.30 & E07 & Barnet & 13 & 79.1 & 80.9 & 0.26 \\
\hline D12 & Suffolk & 17 & 73.4 & 75.7 & 0.37 & $\mathrm{H} 19$ & Kingston and Richmond & 4 & 79.4 & 80.8 & 0.23 \\
\hline $\mathrm{J} 25$ & North and Mid Hampshire & 27 & 73.1 & 75.7 & 0.45 & $\mathrm{~J} 25$ & North and Mid-Hampshire & 26 & 78.8 & 80.8 & 0.30 \\
\hline L35 & Exeter and North Devon & 12 & 73.5 & 75.7 & 0.35 & $\mathrm{H} 21$ & Eastern Surrey & 27 & 78.8 & 80.8 & 0.33 \\
\hline E06 & South West Hertfordshire & 13 & 73.5 & 75.7 & 0.36 & $\mathrm{~J} 22$ & Southampton \& SW Hants* & 6 & 79.3 & 80.8 & 0.23 \\
\hline F31 & North Essex & 11 & 73.5 & 75.6 & 0.35 & $\mathrm{~J} 41$ & Isle of Wight & 14 & 79.1 & 80.8 & 0.23 \\
\hline E18 & East and North Hertfordshire & 8 & 73.6 & 75.6 & 0.36 & E19 & Brent and Harrow & 5 & 79.3 & 80.7 & 0.20 \\
\hline L51 & Somerset & 10 & 73.5 & 75.6 & 0.32 & D13 & East Norfolk & 19 & 79.0 & 80.7 & 0.27 \\
\hline $\mathrm{H} 22$ & West Sussex & 7 & 73.8 & 75.5 & 0.29 & D12 & Suffolk & 16 & 79.0 & 80.6 & 0.25 \\
\hline D13 & East Norfolk & 18 & 73.4 & 75.5 & 0.31 & M20 & Solihull & 9 & 79.2 & 80.6 & 0.21 \\
\hline $\mathrm{K} 24$ & Buckinghamshire & 14 & 73.5 & 75.4 & 0.33 & E18 & East and North Hertfordshire & 20 & 78.9 & 80.6 & 0.26 \\
\hline L40 & Gloucestershire & 33 & 72.9 & 75.4 & 0.41 & M02 & Herefordshire & 40 & 78.3 & 80.6 & 0.35 \\
\hline H19 & Kingston and Richmond & 22 & 73.3 & 75.4 & 0.33 & G21 & East Sussex & 25 & 78.9 & 80.6 & 0.27 \\
\hline M02 & Herefordshire & 9 & 73.5 & 75.3 & 0.30 & E06 & South West Hertfordshire & 33 & 78.6 & 80.6 & 0.30 \\
\hline $\mathrm{J} 22$ & Southampton \& SW Hants* & 25 & 73.2 & 75.3 & 0.33 & L36 & Plymouth and Torbay & 24 & 78.9 & 80.6 & 0.25 \\
\hline $\mathrm{K} 13$ & Berkshire & 26 & 73.1 & 75.3 & 0.35 & E05 & North West Hertfordshire & 12 & 79.1 & 80.6 & 0.23 \\
\hline L10 & Bristol & 29 & 73.0 & 75.2 & 0.35 & L40 & Gloucestershire & 15 & 79.0 & 80.5 & 0.23 \\
\hline $\mathrm{J} 30$ & Wiltshire and Bath & 21 & 73.3 & 75.2 & 0.30 & L10 & Bristol & 32 & 78.6 & 80.5 & 0.30 \\
\hline E09 & Hillingdon & 23 & 73.2 & 75.1 & 0.32 & D09 & Huntingdon & 31 & 78.6 & 80.5 & 0.28 \\
\hline E19 & Brent and Harrow & 19 & 73.4 & 75.1 & 0.27 & F31 & North Essex & 21 & 78.9 & 80.4 & 0.25 \\
\hline M04 & Worcester & 30 & 73.0 & 75.0 & 0.36 & E21 & Ken, Chelsea \& Westmin & 29 & 78.7 & 80.4 & 0.28 \\
\hline $\mathrm{L} 21$ & Cornwall and Isles of Scilly & 37 & 72.8 & 75.0 & 0.37 & $\mathrm{~J} 30$ & Wiltshire and Bath & 28 & 78.7 & 80.3 & 0.24 \\
\hline D09 & Huntingdon & 24 & 73.2 & 75.0 & 0.33 & L21 & Cornwall and Isles of Scilly & 30 & 78.6 & 80.3 & 0.24 \\
\hline B21 & North Yorkshire & 38 & 72.6 & 74.9 & 0.38 & K13 & Berkshire & 41 & 78.3 & 80.1 & 0.28 \\
\hline H09 & Croydon & 50 & 72.3 & 74.9 & 0.41 & E09 & Hillingdon & 10 & 79.2 & 80.1 & 0.15 \\
\hline G21 & East Sussex & 28 & 73.0 & 74.9 & 0.30 & M04 & Worcester & 48 & 78.2 & 80.1 & 0.30 \\
\hline G23 & West Kent & 45 & 72.4 & 74.8 & 0.39 & F32 & South Essex & 42 & 78.3 & 80.1 & 0.27 \\
\hline L36 & Plymouth and Torbay & 32 & 72.9 & 74.8 & 0.30 & G22 & East Kent & 47 & 78.2 & 80.1 & 0.29 \\
\hline G22 & East Kent & 49 & 72.4 & 74.7 & 0.41 & B21 & North Yorkshire & 37 & 78.4 & 80.1 & 0.26 \\
\hline $\mathrm{C} 03$ & Leicestershire & 35 & 72.8 & 74.7 & 0.32 & $\mathrm{~K} 24$ & Buckinghamshire & 22 & 78.9 & 80.1 & 0.20 \\
\hline D05 & North West Anglia & 41 & 72.6 & 74.6 & 0.36 & F34 & Redbridge \& Walth Forest $\ddagger$ & 11 & 79.2 & 80.0 & 0.13 \\
\hline K33 & Northamptonshire & 39 & 72.6 & 74.6 & 0.32 & $\mathrm{C} 03$ & Leicestershire & 38 & 78.4 & 79.9 & 0.24 \\
\hline F32 & South Essex & 34 & 72.8 & 74.6 & 0.32 & F36 & New River & 43 & 78.3 & 79.9 & 0.26 \\
\hline M28 & Warwickshire & 54 & 72.1 & 74.6 & 0.43 & $\mathrm{~J} 21$ & Portsmouth \& SE Hants $†$ & 18 & 79.0 & 79.9 & 0.14 \\
\hline D14 & Bedfordshire & 43 & 72.5 & 74.6 & 0.33 & M05 & Shropshire & 62 & 77.6 & 79.8 & 0.35 \\
\hline $\mathrm{J} 21$ & Portsmouth \& SE Hants $\dagger$ & 40 & 72.6 & 74.6 & 0.33 & D05 & North West Anglia & 49 & 78.1 & 79.8 & 0.28 \\
\hline $\mathrm{J} 41$ & Isle of Wight & 31 & 72.9 & 74.5 & 0.25 & H09 & Croydon & 54 & 77.8 & 79.8 & 0.29 \\
\hline M05 & Shropshire & 48 & 72.4 & 74.5 & 0.36 & G24 & Bexley and Greenwich & 36 & 78.4 & 79.8 & 0.20 \\
\hline M26 & North Worcestershire & 53 & 72.2 & 74.5 & 0.39 & D14 & Bedfordshire & 44 & 78.2 & 79.8 & 0.21 \\
\hline N18 & South Cheshire & 52 & 72.2 & 74.4 & 0.37 & M18 & Dudley & 56 & 77.7 & 79.7 & 0.27 \\
\hline F34 & Redbridge \& Walth Forest $\ddagger$ & 36 & 72.8 & 74.3 & 0.25 & E20 & Ealing, H'smith \& Houns ${ }^{\star \star}$ & 52 & 78.0 & 79.7 & 0.26 \\
\hline $\mathrm{C} 15$ & Lincolnshire & 47 & 72.4 & 74.3 & 0.34 & G23 & West Kent & 39 & 78.4 & 79.6 & 0.20 \\
\hline F36 & New River & 42 & 72.5 & 74.3 & 0.30 & $\mathrm{H} 17$ & Merton, Sutton \& Wands $\oint$ & 35 & 78.5 & 79.6 & 0.18 \\
\hline G24 & Bexley and Greenwich & 46 & 72.4 & 74.3 & 0.33 & P28 & Morecambe Bay & 58 & 77.7 & 79.5 & 0.28 \\
\hline B11 & East Riding & 67 & 71.5 & 74.2 & 0.47 & B61 & Leeds & 74 & 77.2 & 79.5 & 0.35 \\
\hline M27 & South Staffordshire & 56 & 72.0 & 74.2 & 0.34 & M28 & Warwickshire & 65 & 77.5 & 79.5 & 0.31 \\
\hline M18 & Dudley & 64 & 71.5 & 74.2 & 0.46 & N18 & South Cheshire & 61 & 77.6 & 79.4 & 0.28 \\
\hline P16 & Stockport & 57 & 71.9 & 74.2 & 0.38 & F33 & Barking and Havering & 46 & 78.2 & 79.4 & 0.19 \\
\hline F33 & Barking and Havering & 51 & 72.3 & 74.2 & 0.29 & $\mathrm{C} 12$ & Sheffield & 70 & 77.3 & 79.4 & 0.30 \\
\hline P28 & Morecambe Bay & 60 & 71.8 & 74.0 & 0.37 & $\mathrm{C} 15$ & Lincolnshire & 45 & 78.2 & 79.4 & 0.19 \\
\hline $\mathrm{H} 17$ & Merton, Sutton \& Wands $\$$ & 44 & 72.5 & 74.0 & 0.28 & B11 & East Riding & 50 & 78.0 & 79.3 & 0.24 \\
\hline $\mathrm{C} 14$ & North Nottinghamshire & 58 & 71.9 & 74.0 & 0.34 & M27 & South Staffordshire & 67 & 77.4 & 79.3 & 0.29 \\
\hline P22 & South Lancashire & 63 & 71.6 & 73.9 & 0.43 & $\mathrm{C} 08$ & Nottingham & 63 & 77.6 & 79.3 & 0.27 \\
\hline $\mathrm{C} 01$ & North Derbyshire & 55 & 72.1 & 73.9 & 0.30 & $\mathrm{C} 02$ & Southern Derbyshire & 66 & 77.5 & 79.3 & 0.26 \\
\hline $\mathrm{C} 02$ & Southern Derbyshire & 66 & 71.5 & 73.9 & 0.42 & P16 & Stockport & 60 & 77.7 & 79.2 & 0.26 \\
\hline B61 & Leeds & 72 & 71.2 & 73.9 & 0.44 & M26 & North Worcestershire & 53 & 77.9 & 79.2 & 0.21 \\
\hline $\mathrm{C} 08$ & Nottingham & 59 & 71.8 & 73.6 & 0.32 & M22 & Wolverhampton & 72 & 77.2 & 79.2 & 0.28 \\
\hline B16 & Grimsby and Scunthorpe & 65 & 71.5 & 73.5 & 0.32 & K33 & Northamptonshire & 51 & 78.0 & 79.1 & 0.15 \\
\hline N43 & Sefton & 71 & 71.2 & 73.5 & 0.38 & $\mathrm{C} 14$ & North Nottinghamshire & 69 & 77.3 & 79.1 & 0.29 \\
\hline A11 & Northumberland & 69 & 71.3 & 73.5 & 0.41 & B16 & Grimsby and Scunthorpe & 78 & 77.1 & 79.0 & 0.28 \\
\hline E21 & Ken, Chelsea \& Westmin & 68 & 71.3 & 73.5 & 0.34 & M25 & South Birmingham & 81 & 77.0 & 79.0 & 0.32 \\
\hline N51 & Wirral & 70 & 71.2 & 73.4 & 0.36 & N51 & Wirral & 71 & 77.3 & 78.9 & 0.20 \\
\hline A34 & North Cumbria & 74 & 71.0 & 73.3 & 0.38 & $\mathrm{C} 01$ & North Derbyshire & 68 & 77.4 & 78.9 & 0.21 \\
\hline $\mathrm{C} 12$ & Sheffield & 62 & 71.7 & 73.3 & 0.30 & F37 & Camden and Islington & 57 & 77.7 & 78.8 & 0.20 \\
\hline B51 & West Yorkshire & 86 & 70.7 & 73.2 & 0.45 & N43 & Sefton & 64 & 77.6 & 78.8 & 0.22 \\
\hline E20 & Ealing, H'smith \& Houns ${ }^{\star \star}$ & 61 & 71.7 & 73.2 & 0.25 & P22 & South Lancashire & 82 & 76.9 & 78.8 & 0.32 \\
\hline
\end{tabular}

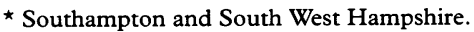

+ Portsmouth and South East Hampshire.

$\ddagger$ Redbridge and Waltham Forest.

$\delta$ Merton, Sutton and Wandsworth.

I Kensington, Chelsea and Westminster.

$\star \star$ Ealing, Hammersmith and Hounslow. 


\begin{tabular}{|c|c|c|c|c|c|c|c|c|c|c|c|}
\hline \multirow[b]{2}{*}{ Males } & & \multirow{2}{*}{$\begin{array}{l}\text { Ranks } \\
\text { in } \\
1984-86\end{array}$} & \multicolumn{2}{|c|}{ Life expectancy } & \multirow{2}{*}{$\begin{array}{l}\% \\
\text { annual } \\
\text { increase }\end{array}$} & & & \multirow{2}{*}{$\begin{array}{l}\text { Ranks } \\
\text { in } \\
1984-86\end{array}$} & \multicolumn{2}{|c|}{ Life expectancy } & \multirow{2}{*}{$\begin{array}{l}\% \\
\text { annual } \\
\text { increase }\end{array}$} \\
\hline & & & $1984-86$ & $1992-94$ & & \multicolumn{2}{|c|}{ Females } & & $1984-86$ & $1992-94$ & \\
\hline $\mathrm{C} 10$ & Doncaster & 75 & 71.0 & 73.2 & 0.38 & A34 & North Cumbria & 80 & 77.0 & 78.8 & 0.29 \\
\hline M21 & Walsall & 81 & 70.8 & 73.1 & 0.36 & M17 & Coventry & 59 & 77.7 & 78.7 & 0.17 \\
\hline C11 & Rotherham & 73 & 71.2 & 73.1 & 0.29 & P20 & North West Lancashire & 77 & 77.2 & 78.7 & 0.24 \\
\hline B31 & Bradford & 96 & 70.3 & 72.9 & 0.43 & $\mathrm{C} 11$ & Rotherham & 76 & 77.2 & 78.6 & 0.23 \\
\hline B71 & Wakefield & 87 & 70.6 & 72.9 & 0.42 & $\mathrm{C} 10$ & Doncaster & 79 & 77.1 & 78.5 & 0.25 \\
\hline M17 & Coventry & 78 & 70.9 & 72.9 & 0.34 & B51 & West Yorkshire & 97 & 76.3 & 78.5 & 0.33 \\
\hline A31 & South Durham & 91 & 70.5 & 72.9 & 0.39 & B31 & Bradford & 96 & 76.3 & 78.5 & 0.33 \\
\hline P23 & Wigan and Bolton & 100 & 70.2 & 72.8 & 0.44 & G26 & South East London & 55 & 77.7 & 78.4 & 0.11 \\
\hline N17 & North Cheshire & 77 & 71.0 & 72.8 & 0.32 & M07 & North Staffordshire & 87 & 76.5 & 78.4 & 0.29 \\
\hline M07 & North Staffordshire & 92 & 70.4 & 72.8 & 0.41 & A11 & Northumberland & 89 & 76.5 & 78.3 & 0.28 \\
\hline P20 & NW Lancashire & 83 & 70.7 & 72.7 & 0.30 & M29 & North Birmingham & 83 & 76.8 & 78.3 & 0.24 \\
\hline $\mathrm{C} 09$ & Barnsley & 76 & 71.0 & 72.7 & 0.29 & M19 & Sandwell & 73 & 77.2 & 78.3 & 0.20 \\
\hline M29 & North Birmingham & 89 & 70.6 & 72.6 & 0.34 & A31 & South Durham & 86 & 76.7 & 78.1 & 0.23 \\
\hline M25 & South Birmingham & 82 & 70.8 & 72.6 & 0.32 & M21 & Walsall & 91 & 76.4 & 78.1 & 0.28 \\
\hline M22 & Wolverhampton & 79 & 70.9 & 72.6 & 0.30 & $\mathrm{C} 09$ & Barnsley & 88 & 76.5 & 78.1 & 0.23 \\
\hline A35 & Newcastle and North Tyneside & 84 & 70.7 & 72.6 & 0.29 & A35 & Newcastle and North Tyneside & 84 & 76.8 & 78.1 & 0.20 \\
\hline A 30 & North Durham & 93 & 70.4 & 72.6 & 0.36 & F35 & East London and the City & 75 & 77.2 & 78.1 & 0.17 \\
\hline P24 & Bury and Rochdale & 90 & 70.5 & 72.5 & 0.35 & N17 & North Cheshire & 98 & 76.2 & 78.0 & 0.30 \\
\hline P21 & East Lancashire & 94 & 70.4 & 72.4 & 0.38 & B71 & Wakefield & 94 & 76.3 & 78.0 & 0.26 \\
\hline P27 & Salford and Trafford & 95 & 70.3 & 72.4 & 0.36 & P27 & Salford and Trafford & 85 & 76.7 & 78.0 & 0.20 \\
\hline N31 & St Helens and Knowsley & 102 & 70.0 & 72.4 & 0.41 & P24 & Bury and Rochdale & 95 & 76.3 & 77.9 & 0.22 \\
\hline A32 & Tees & 99 & 70.3 & 72.2 & 0.34 & A 30 & North Durham & 93 & 76.4 & 77.8 & 0.20 \\
\hline A16 & Sunderland & 97 & 70.3 & 72.1 & 0.32 & P23 & Wigan and Bolton & 100 & 76.2 & 77.8 & 0.28 \\
\hline P25 & West Pennine & 88 & 70.6 & 72.1 & 0.27 & P21 & East Lancashire & 104 & 75.9 & 77.8 & 0.33 \\
\hline M19 & Sandwell & 101 & 70.2 & 72.0 & 0.31 & A33 & South of Tyne & 90 & 76.5 & 77.8 & 0.22 \\
\hline A33 & South of Tyne & 103 & 70.0 & 71.9 & 0.34 & A32 & Tees & 92 & 76.4 & 77.8 & 0.23 \\
\hline F37 & Camden and Islington & 80 & 70.9 & 71.8 & 0.16 & P25 & West Pennine & 99 & 76.2 & 77.7 & 0.24 \\
\hline G26 & South East London & 85 & 70.7 & 71.7 & 0.18 & N31 & St Helens and Knowsley & 102 & 76.1 & 77.6 & 0.24 \\
\hline F35 & East London and the City & 98 & 70.3 & 71.7 & 0.23 & A16 & Sunderland & 101 & 76.2 & 77.4 & 0.17 \\
\hline $\mathrm{N} 21$ & Liverpool & 104 & 69.8 & 71.2 & 0.24 & $\mathrm{~N} 21$ & Liverpool & 103 & 76.0 & 77.3 & 0.21 \\
\hline P26 & Manchester & 105 & 69.2 & 69.9 & 0.10 & P26 & Manchester & 105 & 75.4 & 76.7 & 0.22 \\
\hline
\end{tabular}

Table 3 Life expectancy at birth in relation to gender in 1984-86 and 1992-94: English district health authorities grouped by level of deprivation, listed in order of increasing deprivation

\begin{tabular}{|c|c|c|c|c|c|c|c|c|c|c|}
\hline \multirow{3}{*}{$\begin{array}{l}\text { Level of } \\
\text { deprivation }\end{array}$} & \multicolumn{4}{|l|}{ Males } & \multicolumn{4}{|l|}{ Females } & \multirow{2}{*}{\multicolumn{2}{|c|}{$\begin{array}{l}\text { Female-male } \\
\text { difference in life } \\
\text { expectancy (y) }\end{array}$}} \\
\hline & \multirow{2}{*}{$\begin{array}{l}\text { Rank in } \\
1984-86\end{array}$} & \multicolumn{2}{|c|}{ Life expectancy } & \multirow{2}{*}{$\begin{array}{l}\% \\
\text { annual } \\
\text { increase }\end{array}$} & \multirow{2}{*}{$\begin{array}{l}\text { Rank in } \\
1984-86\end{array}$} & \multicolumn{2}{|c|}{ Life expectancy } & \multirow{2}{*}{$\begin{array}{l}\% \\
\text { annual } \\
\text { increase }\end{array}$} & & \\
\hline & & $1984-86$ & $1992-94$ & & & $1984-86$ & $1992-94$ & & $1984-86$ & $1992-94$ \\
\hline $\begin{array}{l}\text { England } \\
\text { Deprivation }\end{array}$ & & 72.1 & 74.1 & 0.35 & & 77.9 & 79.5 & 0.25 & 5.8 & 5.4 \\
\hline $\begin{array}{c}\text { level } 1 \\
\text { Deprivation }\end{array}$ & 1 & 73.0 & 75.2 & 0.38 & 2 & 78.4 & 80.2 & 0.28 & 5.4 & 4.9 \\
\hline $\begin{array}{c}\text { level } 2 \\
\text { Deprivation }\end{array}$ & 2 & 72.9 & 75.0 & 0.35 & 1 & 78.5 & 80.1 & 0.25 & 5.5 & 5.1 \\
\hline $\begin{array}{c}\text { level } 3 \\
\text { Deprivation }\end{array}$ & 3 & 72.6 & 74.8 & 0.36 & 3 & 78.4 & 80.0 & 0.25 & 5.8 & 5.3 \\
\hline $\begin{array}{c}\text { level } 4 \\
\text { Deprivation }\end{array}$ & 4 & 71.6 & 73.7 & 0.35 & 4 & 77.6 & 79.1 & 0.24 & 6.0 & 5.5 \\
\hline $\begin{array}{c}\text { level } 5 \\
\text { Deprivation }\end{array}$ & 5 & 71.1 & 73.0 & 0.34 & 6 & 77.1 & 78.7 & 0.25 & 6.0 & 5.6 \\
\hline $\begin{array}{c}\text { level } 6 \\
\text { Deprivation }\end{array}$ & 6 & 71.0 & 72.9 & 0.32 & 5 & 77.3 & 78.9 & 0.26 & 6.3 & 6.0 \\
\hline level 7 & 7 & 70.2 & 71.2 & 0.18 & 7 & 76.8 & 77.8 & 0.17 & 6.7 & 6.6 \\
\hline
\end{tabular}

Note: definition of deprivation levels is given in table 1 .

0.45 years (table 3 ). Affluent areas showed a greater narrowing of this differential than deprived areas: -0.48 years in the most affluent areas, with practically no change in the most deprived areas $(-0.06$ years $)$.

\section{Discussion}

There have been major changes in ONS systems of processing mortality data, which came into effect from 1993 and affect cause of death coding. The all cause mortality data used here are unaffected, except for the change from numbers of registrations annually to numbers of occurrences. ONS expects this change to have little effect on annual totals.

Life expectancy conveys the impact of mortality more readily than rates/ratios. It should, however, be noted that life table methodology is based on death rates prevailing in a particular period, comprising the mortality experience of many successive birth cohorts Hence life expectancy is a "period" measure, describing how long a person would live if the mortality rates of a particular period prevailed for an entire generation. In this paper we have measured life expectancy based on mortality levels in the decade up to 1994; since it is realistic to expect mortality to continue to fall in the future, those born now are likely to live longer than predicted by this approach.

The findings presented here quantify the effects on longevity of regional differences in mortality in England. For instance, given the local mortality rates in the early 1990 s, a male baby in Cambridge can expect to live almost 7 years longer than his counterpart in Manchester, the difference for a female baby being almost 4.5 years. Furthermore, regional differences have widened over the past decade. The lowest ranking areas in this analysis have 

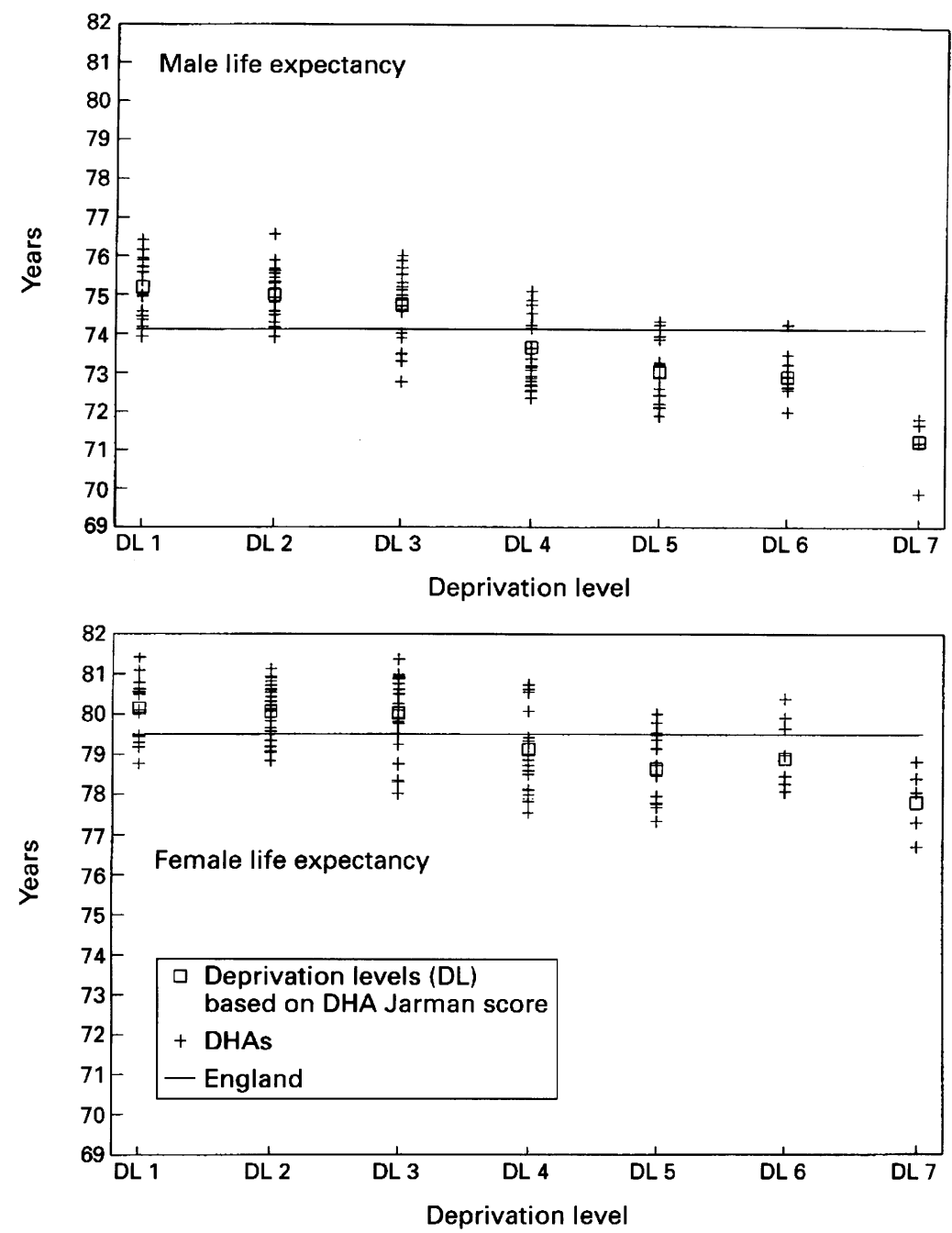

Figure 3 Male and female life expectancy at birth in district health authorities in England, 1992-94. Note: $D L 1<-S D$ (ie $<-17.37) ; D L 2$, between -1 and -0.5 of the $S D ; D L 3$, between -0.5 and 0 of the $S D ; D L 4$, between 0 and 0.5 of the $S D ; D L 5$, between 0.5 and 1 of the $S D ; D L 6$, between 1 and 1.96 of the $S D ; D L 7$, significantly greater than the mean at the $5 \%$ level.

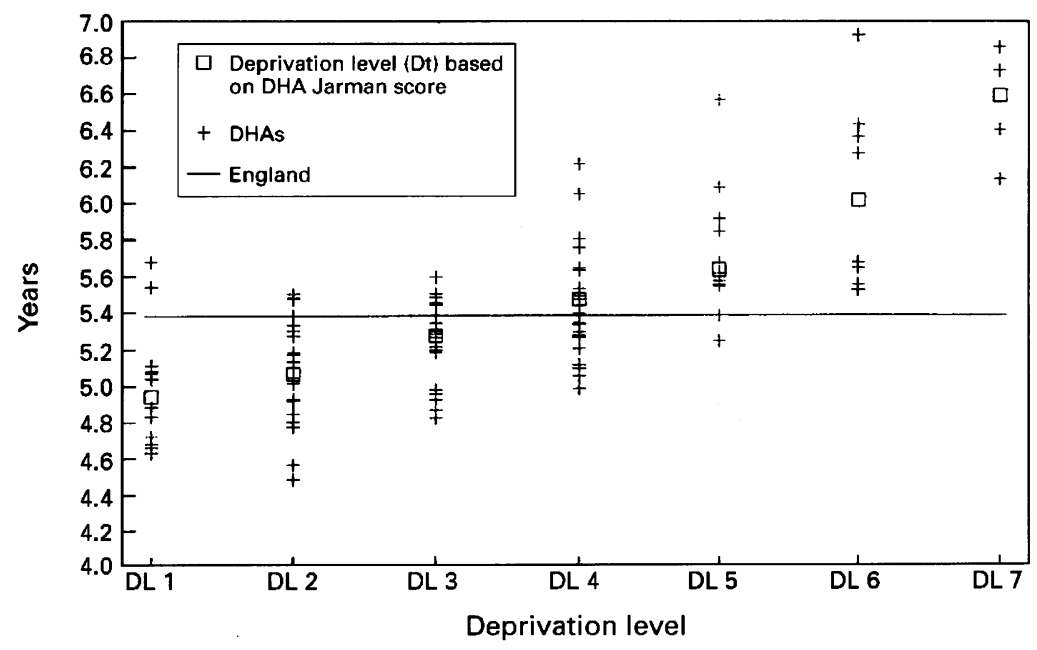

Figure 4 Female-male differences in life expectancy among English district health authorities, 1992-94. Note: deprivation levels are defined in the same way as in figure 3.

life expectancies in 1992-94 equivalent to those of England and Wales two decades ago. ${ }^{3}$

Using Jarman scores ${ }^{6}$ as a measure of deprivation, we found a correlation coefficient between deprivation and life expectancy of -0.77 for men and -0.56 for women. Although other measures of deprivation are also available, ${ }^{1011}$ Jarman scores were used here because they are available for the 1995 DHA boundaries. Any method combining several variables into a single index has its limitations. However, correlation coefficients obtained by Eames et al between mortality and different deprivation indices do not vary greatly, ${ }^{12}$ and are similar to our results.

We have shown that not only is life expectancy in English DHAs inversely associated with deprivation, but that the prosperous, longest lived populations have seen the greatest gains in life expectancy over the decade. In contrast, the most deprived DHAs (inner London, Manchester and Liverpool) experienced negligible improvements in longevity, despite having the lowest life spans in the mid-1980s. Inner London has shown the poorest gains, and male life expectancy in parts of the capital is among the lowest in the country. Another significant finding is that survival rates for young men aged 30-39 have deteriorated nationally, and in all area groupings, the decline being greatest in deprived areas, which also showed a significant fall in survival rates at 20-29. Mortality increased also in young women, but only in the very deprived areas.

From the data available, it is not possible to quantify the contribution of migration to life expectancy variations between DHAs. It is possible that the higher life expectancy of an area reflects in-migration of healthy people (and conversely), and that widening geographical differences reflect, at least in part, the effects of selective migration. While the health of migrants has important policy implications, such data would be available only on a sample basis. Furthermore, it does not detract from the value of an ecological investigation into local variations in health in order to identify the most disadvantaged areas.

The findings presented here are consistent with those noted by Charlton in his analysis of life expectancy for regional health authorities and the ONS area classification groups. ${ }^{5}$ The latter is an area typology based on homogeneity across a range of demographic, housing, and socioeconomic variables. Charlton went on to show that the high mortality "Ports and industry" areas have the highest mortality from lung cancer, coronary heart disease, and stroke, and that people in inner London have the highest mortality from respiratory diseases and injury and poisoning.

There is mounting evidence about the association between socioeconomic inequalities and mortality differentials in England, ${ }^{12-23}$ and that the degree of socioeconomic inequality within an area contributes an additional effect over and above that of the level of deprivation alone. ${ }^{240}$ The analysis of Phillimore et al showed a widening of mortality differentials between affluent and deprived electoral wards in northern England between 1981 and 1991. ${ }^{13}$ Eames et al have shown that premature mortality from all causes, coronary heart disease, and smoking-related diseases is associated with deprivation..$^{12}$ Sloggett and Joshi used longitudinal data to distinguish between the effects on 
mortality of deprivation at population and individual level. ${ }^{23}$ They found that it is disadvantaged people that are at risk, irrespective of whether they live in deprived or affluent areas, and concluded that people not areas should be targeted by health interventions.

In contrast, Illsley and Le Grand concluded that age and sex specific regional trends in mortality between 1931 and 1987-89 suggest that behavioural risk factors and ethnicity, rather than deprivation, make the greatest contribution to the persistence of geographical differentials. ${ }^{31}$ They noted that a convergence in regional differences in childhood mortality, the most vulnerable to the effects of poverty, contrasted with widening differences among the middle aged after 1961, which they attributed to differential changes in risk behaviour relating to coronary heart disease and lung cancer, with affluent groups responding most effectively to health promotion messages about behaviour induced diseases.

Although the major impact on life expectancy of regional differences in mortality is registered in middle aged adults, a significant difference in mortality continues to be apparent even at younger ages. This is reflected in the 1993 SMRs for ages 0-14 years of 72 in prosperous areas and 89 in growth areas, contrasting with 125 in manufacturing areas and 116 in inner London (England and Wales $=100) .^{7}$ Thus, although the historical narrowing of mortality differentials regionally has been greatest at younger ages, the differences remain substantial even now.

However, Illsley and Le Grand's argument is consistent with our findings that regional differences in life expectancy are greater for males than for females, and that deprived areas not only have lower life expectancies but also greater gender differences. Deprived areas also showed the least narrowing of gender differences over the decade. It is not clear why deprivation should impact more on male than on female longevity. If the higher mortality of people in deprived areas reflects the cumulative effects of deprivation through childhood and later life, ${ }^{30}{ }^{32}$ it is not obvious why the effects should be greater for men than for women. Possible explanations are: selective migration to deprived areas of males in poor health; greater gender variation in deprived areas in the incidence of external causes of death such as accidents, suicide and violence; and finally, deprivation could be a stronger proxy for health risk behaviour in men than in women.

Rising mortality in young men irrespective of the area's socioeconomic status may reflect the increasing contribution of suicide and/or AIDS; the adverse effects on survival among young women in very deprived areas is less readily explained. Government population projections for England anticipated an AIDS related increase in young male mortality up to the mid-1990s; they also forecast an increase in young female mortality between 1994 and 1995. ${ }^{33}$

Areas with low life expectancy (particularly inner London) are also those with substantial ethnic minority populations. Infant mortality is significantly higher than the national average in infants of African-Caribbean and Pakistani born mothers, but not significantly different in infants of mothers born in India, Bangladesh or East Africa. ${ }^{34}$ The 1991 census based analysis of mortality in England and Wales by country of birth shows raised SMRs at ages 20-69 for migrants from Africa and for South Asian men (but not women); Caribbean migrants had low SMRs. ${ }^{35}$ The contribution of ethnicity to the regional differences in life expectancy noted here is difficult to ascertain but, since ethnicity is associated with deprivation, it is unlikely to have a strong independent effect.

The congruence between behavioural risk factors, the insults to physical and mental health imposed by deprivation, and ethnicity makes the issue of causation particularly complex. The Variations In Health report ${ }^{2}$ concluded that differential lifetime exposure to health damaging or health promoting physical and social environments is the main explanation for variations in life expectancy, with health related social mobility, health damaging or health promoting behaviours, use of health services, and biological factors also contributing. In the next phase of this work we will explore shifts in age, gender and cause specific mortality to help identify the nature and sources of the variations noted in this paper.

Funding: none.

Conflicts of interest: none.

Secretary of State for Health. The health of the nation: a strategy for health in England. London: HMSO, 1992.

Department of Health. The health of the nation. Variations in health: what can the Department of Health and the NHS do? A report produced by the Variations Sub-Group of the Chief Medical Officer's Health of the Nation Working group. London: HMSO, 1995.

3 OPCS. Mortality statistics: England and Wales. Series DH1 no.24. London: HMSO, 1991

4 Gardner MJ, Donnan SPB. Life expectancy: variations among regional health authorities. Population Trends 1977;10:10-2.

5 Charlton J. Which areas are the healthiest? Population Trends 1996;83:17-24.

6 Jarman B. Underprivileged areas: validation and distribution of scores. BMF 1984;289:1587-92.

7 Department of Health/University of Surrey. Public Health Common Data Set 1996: England, Volumes I, II, III and IV. Guildford: National Institute of Epidemiology, University of Surrey, 1997

8 Elandt-Johnson RC, Johnson NL. Survival models and data analysis. New York: John Wiley, 1980.

OPCS. Deaths in 1993 by cause: provisional numbers. OPCS Monitor DH2 94/2. London: HMSO, 1994.

10 Carstairs V, Morris R. Deprivation and health in Scotland. Aberdeen: Aberdeen University Press, 1991

11 Townsend P, Phillimore P, Beattie A. Health and deprivation. Inequality and the north. London: Croom Helm, 1988.

12 Eames M, Ben-Shlomo Y, Marmot MG. Social deprivation and premature mortality: regional comparison across Engand. $B M 7$ 1993:307:1097-102

13 Phillimore P, Beattie A, Townsend P. Widening inequality of health in northern England, 1981-91. BMJ 1994;308 $1125-8$

14 Townsend $\mathrm{P}$, Davidson $\mathrm{N}$. Inequalities in health Harmondsworth: Penguin Books, 1982

15 Black D. Inequalities in health. Public Health 1991;105:23-7.

6 Townsend P, Davidson N, Whitehead M. Inequalities in health: the Black report and the health divide. Harmondsworth: Penguin Books, 1988.

17 Balarajan R, Yuen P, Machin D. Inequalities in health changes in RHAs in the past decade. BM7 1987;294:1561

18 Marmot MG, McDowall ME. Mortality decline and widening social inequalities. Lancet 1986:ii:274-6.

9 Morris JN. Inequalities in health: ten years and little further on. Lancet 1990;336:491-3.

20 Wilkinson RG. Class mortality differentials, income distribution and trends in poverty 1921-81. Fournal of Social bution and trends in
Policy 1989;18:307-35.

21 Davey Smith G, Morris J. Increasing inequalities in the health of the nation. BMF 1994;309:1453-4. 
22 Mackenbach JP. Tackling inequalities in health. BMf 1995; 310:1 152-3.

23 Slogget $A$, Joshi $H$. Higher mortality in depived areas: community or personal disadvantage? $B M \mathcal{F}$ 1994;309: 1470-4.

24 Wilkinson RG. Income distribution and life expectancy.

25 Wilkinson RG. Divided we fall: the poor pay the price of increased social inequality with their health. BMF 1994; 308:1113-4.

26 Wilkinson RG. Commentary: a reply to Ken Judge: mistaken criticisms ignore overwhelming evidence. $B M \mathcal{F}$ 1995;311:1285-7.

27 Ben-Shlomo Y, White IR, Marmot M. Does the variation in the socioeconomic characteristics of an area affect mortality? BMF 1996;312:1013-4.

28 Kaplan GA, Pamuk ER, Lynch JW, Cohen RD, Balfour JL. Inequality in income and mortality in the United States: analysis of mortality and potential pathways. BMF 1996; 312:999-1003.
29 Kennedy BP, Kawachi I, Prothrow-Stith D. Income distribution and mortality: cross sectional ecological stud 312:1004-7. Davey Smith G. Income inequality

they related? $B M f 1996 ; 312: 987-8$.
Illsley $R$, Le Grand J. Regional inequalities in mortality. $\mathcal{f}$ Epidemiol Community Health 1993;47:444-9.

32 Watt GCM. All together now: why social deprivation matters to everyone. $B M \mathcal{F} 1996 ; 312 ; 1026-9$.

33 Government Actuary's Department. Population projections by the government actuary: 1994 based principal projection. London: Government Actuary's Department, 1996.

34 Soni Raleigh V, Balarajan R. The health of infants and children among ethnic minorities. In: Botting $\mathrm{B}$, ed. The health of our children. OPCS Decennial Supplement, series noll. London: HMSO, 1995.

35 Wild S, McKeigue P. Cross sectional analysis of mortality by country of birth in England and Wales, 1970-92. BMF 1997;314:705-10. 